\title{
A multiscale modeling approach to glioma invasion with therapy
}

\author{
Alexander Hunt and Christina Surulescu
}

April 8, 2015

\begin{abstract}
We consider the multiscale model for glioma growth introduced in [19] and extend it to account for therapy effects. Thereby, three treatment strategies involving surgical resection, radio-, and chemotherapy are compared for their efficiency. The chemotherapy relies on inhibiting the binding of cell surface receptors to the surrounding tissue, which impairs both migration and proliferation.
\end{abstract}

\section{Introduction}

Malignant gliomas are highly invasive and heterogeneous primary brain tumors. Their treatment is still elusive, in spite of the development and diversification of the therapeutic approaches [48]. The differentiated response of tumor cell subpopulations to treatment and the infiltrative growth throughout the brain tissue $[23,37,39]$ are two main causes for the lack of surgical, radio- and chemotherapeutical cure. Indeed, it is largely accepted that cells with a highly proliferating phenotype are more sensitive to both chemo and radiotherapy, whereas the migratory phenotype is attended by reduced treatment sensitivity, see e.g., [39, 42,60] and the references therein. Moreover, because of the high affinity of glioma cells to myelinated fiber tracts in the white matter [24, 26], in more than $90 \%$ of cases, the recurrent tumor develops immediately adjacent to the resection margin or within several centimeters of the resection cavity [23, 39].

In this work we address both the modeling of (infiltrative) glioma spread and growth, and the response to surgical resection and radiotherapy. Concerning the treatment we use the idea of reducing tumor cell migration by inhibiting the binding of cell surface receptors to the tissue fibers in the peritumoral region (see e.g., [11, 23] and the references therein), hence also rendering the cancer cells more sensitive against radiotherapy, in view of the go-or-grow hypothesis stating that the tumor cells can either migrate or proliferate $[9,25,23,29]$. On the other hand, however, the receptor binding inhibition can also impair cell proliferation, as the latter is known to be influenced by cell-matrix (and cell-cell) adhesion [14, 27, 32, 40, 45, 63]; hence, the balance between increasing proliferation through stopping migration and reducing mitotic activity through inhibiting adhesion will be the driving factor for enhancing radiosensitivity. Mathematical models for the therapy of glioma have also been considered in $[2,51,52]$, however in a much simplified monoscale case not able to account for the highly infiltrative behavior of this type of cancer. Here we start from the multiscale setting introduced in [19] to describe the evolution of a heterogeneous tumor consisting of migrating and proliferating glioma cells moving along white matter tracts of white matter. The anisotropic structure of the brain is assessed from diffusion tensor imaging (DTI) data and the model involves on the microscopic, subcellular scale the receptor binding dynamics to the tissue fibers, while the individual cell dynamics are modeled on the mesoscale via kinetic transport equations. A parabolic scaling allows to deduce effective equations for the tumor growth on the macroscopic (population) scale, carrying the information from the lower scales. This model permits to account for the infiltrative behavior of glioma and in this work we extend it to also consider therapy in the sense stated above. Another multiscale model involving intracellular (microscale) and extracellular $\mathrm{pH}$ dynamics along with the evolution of tumor cells and normal tissue (macroscale) has been proposed in [61] and extended in [44] to include the issue of treatment with sensitization against radiotherapy via alkalinization of the tumor microenvironment and depletion of cancer cells via chemotherapy and radiotherapy.

This paper is organized as follows: In Section 2 we present the mathematical model and describe 
the way in which therapy is considered and implemented, then use a parabolic scaling to re-deduce (with the changes due to modeling therapy) the effective equations for glioma spread involving the DTI information for the patient specific brain structure and encompassing the subcellular receptor binding dynamics in the diffusion, transport and haptotaxis coefficients. The well posedness of both formulations (multiscale and population level, respectively) is addressed as well. Section 3 is concerned with the assessment of the functions and parameters employed in the model, and Section 4 presents the numerical method and the simulations obtained from our setting. Eventually, Section 5 offers some comments about the potential of this modeling approach and further issues to be addressed in this context.

\section{Mathematical modeling}

\subsection{Equations on the mesoscopic and microscopic levels}

In [19] we considered a model for glioma invasion relying on the go-or-grow hypothesis. In this work we extend that setting in order to account for therapy. Thereby, in view of the migratory/proliferative phenotype of cancer cells significantly influencing the response of tumors to various treatments like radiotherapy and chemotherapy, we will characterize this differentiated response by considering two tumor subpopulations: proliferative (hence non-motile) and migrating cells, respectively.

We consider the density function $p(t, \mathbf{x}, \mathbf{v}, y)$ of moving cells at time $t$ and position $\mathbf{x} \in \mathbb{R}^{n}$, with velocity $\mathbf{v} \in V \subset \mathbb{R}^{n}$ and receptor state $y \in \mathbb{R}_{+}$, and the density function $r(t, \mathbf{x}, y)$ of resting cells. Thereby, the receptor state $y$ is a subcellular scale (microscale) variable and refers to the volume fraction of cell surface receptors bound to insoluble ligands in the surrounding tissue. While it is clear that integrins (a family of heterodimeric cell adhesion molecules playing a crucial role in cell-cell and cell-matrix interaction [32, 33, 45]) and their binding to the ECM are essential for glioma migration [15, 63] and it has been found that glioma cells follow the anisotropic brain structure along the white matter tracts $[24,26]$, the mechanism of adhesion to the myelinated axons is still not elucidated, but integrins do not seem to be responsible for such bindings [24]. In the following we will use the syntagma 'cell surface receptors' for all kinds of receptors involved in cell-tissue adhesion and will concentrate on integrins when referring to chemotherapeutical agents which aim at inhibiting migration and proliferation. For further discussions on the issue of glioma-tissue adhesion we refer to [17, 19].

The two cancer cell densities of interest satisfy on the mesoscale the system of partial integrodifferential equations

$$
\begin{aligned}
\partial_{t} p+\nabla_{\mathbf{x}} \cdot(\mathbf{v} p) & +\partial_{y}\left(G\left(y, Q, d_{c}, d_{r}\right) p\right) \\
& =\mathcal{L}[\lambda(y)] p-a\left(\mathbf{x}, d_{c}\right) p+b\left(\mathbf{x}, d_{c}\right) \frac{q(\hat{\mathbf{v}})}{\omega} r-\left(l_{1}(N)+R_{1}\left(\alpha_{1}, d_{r}\right)\right) p, \\
\partial_{t} r & =a\left(\mathbf{x}, d_{c}\right) \int_{V} p(\mathbf{v}) d \mathbf{v}-b\left(\mathbf{x}, d_{c}\right) r+g\left(N, d_{c}\right) r-\left(l_{2}(N)+R_{2}\left(\alpha_{2}, d_{r}\right)\right) r,
\end{aligned}
$$

where $\mathcal{L}[\lambda(y)] p:=-\lambda(y) p+\lambda(y) \int_{V} K(\mathbf{x}, \mathbf{v}) p\left(\mathbf{v}^{\prime}\right) d \mathbf{v}^{\prime}$ is the turning operator modeling the cell velocity innovations due to contact guidance, environmental cues etc. Here the turning kernel $K$ accounting for such influences is taken for simplicity to be of the form (see [30]) $K(\mathbf{x}, \mathbf{v}):=\frac{q(\hat{\mathbf{v}})}{\omega}$, where $\hat{\mathbf{v}}$ is the normalized velocity, $q(\mathbf{x}, \hat{\mathbf{v}})$ is the directional distribution of tissue fibers, and $\omega=\int_{V} q(\hat{\mathbf{v}}) d \mathbf{v}$ is a scaling constant (we assume $V=s \mathbb{S}^{n-1}$, with $s$ given) such that $K$ is indeed a kernel. The function $\lambda(y)$ denotes as in $[17,19,18]$ the turning rate of the cells. Motivated by existing experimental evidence (see e.g., [46]) that integrins expressed by resting cells cannot in general bind their ligands, we assume this also for our model, as we did in [19]. ${ }^{1}$

In (1) and (4) below $Q(t, \mathbf{x})$ denotes the (macroscopic) volume fraction of tissue (including as in [17, 19] ECM and neuron bundles). The functions $a\left(\mathbf{x}, d_{c}\right)$ and $b\left(\mathbf{x}, d_{c}\right)$ denote the rates with which cells stop and proliferate, respectively start moving after a resting (proliferating) phase. The cells which exit proliferation and become motile are doing this by interacting with the tissue, which motivates the factor $\frac{q(\hat{\mathbf{v}})}{\omega}$ in the corresponding term of (1). Both $a$ and $b$ can depend on the position $\mathbf{x}$ and are supposed to also depend on the dose $d_{c}$ of the chemotherapeutic agent. $g\left(N, d_{c}\right)$ and $l_{i}(N)(i=1,2)$ are functions representing gain and loss due to cell proliferation and death, respectively. Thereby, the

\footnotetext{
${ }^{1}$ In particular, this justifies the omission of the 'transport' term w.r.t. $y$ on the left hand side of (2)
} 
gain is impaired by the effect of chemotherapy and the loss is amplified by radiotherapy. The effects of the latter are described by the terms $R_{j}\left(\alpha_{j}, d_{r}\right)$, where

$$
R_{j}\left(\alpha_{j}, d_{r}\right)=\sum_{i=1}^{\nu}\left(1-S\left(\alpha_{j}, d_{r}\right)\right) \eta_{\delta}\left(t-t_{i}\right), \quad t_{i} \in \text { radiotherapy }
$$

with $j=1,2,3$ and 'radiotherapy' denoting the set of times at which ionizing radiation is applied to the patient (with dose $d_{r}$ ). Here $\nu$ is the number of fractions, $\eta_{\delta}$ is a $C_{0}^{\infty}$ function with unit mass and support in $(-\delta, \delta), \delta<<1$ and $S\left(\alpha_{j}, d_{r}\right)=\exp \left(-\alpha_{j} d_{r}-\beta_{j} d_{r}^{2}\right)$ models the survival fraction of each subpopulation $p, r$ or normal tissue (for $j=3$ ), respectively, after application of radiotherapy with a dose $d_{r}$ (in Gy). Thus, we adopted the linear quadratic (LQ) model [21, 28, 54], which in spite of its shortcomings [10, 35, 65] is still the standard choice in radiation treatments (see e.g., [50, 59]). The parameter $\alpha_{j}$ represents lethal lesions produced by a single radiation track (they are linearly related to the dose: $\alpha_{j} d_{r}$, cell kill per Gy), while $\beta_{j}$ characterizes lethal lesions produced by two radiation tracks (quadratically related to the dose: $\beta_{j} d_{r}^{2}$, cell kill per $\mathrm{Gy}^{2}$ ). The relevant parameter in the LQ model is actually the radiation sensitivity $\frac{\alpha_{j}}{\beta_{j}}$, which correlates to the cell cycle length: late responding tissues with a slow cell cycle have a small $\frac{\alpha_{j}}{\beta_{j}}$ ratio, while it is large for early responding, highly aggressive cancers [59]. In clinical practice the total dose $d_{r}$ is given in $\nu$ fractions of size $\hat{d}_{r}$, hence

$$
S\left(\alpha_{j}, d_{r}\right)=\exp \left(-\nu\left(\alpha_{j} \hat{d}_{r}+\beta_{j} \hat{d}_{r}^{2}\right)\right)=\exp \left(-\alpha_{j} d_{r}\left(1+\hat{d}_{r} /\left(\alpha_{j} / \beta_{j}\right)\right)\right) .
$$

Concerning chemotherapy, we concentrate on reducing invasion and proliferation and not necessarily on achieving cell kill. In our model the latter is supposed to be due to ionizing radiation, however the setting can be easily extended to include a further chemotherapeutic agent ${ }^{2}$ triggering cell death. Most types of cells depend on integrin-mediated adhesion to ECM for migration, proliferation, and survival. In particular, glioma cells have highly migrating potential, which accounts for glioma recurrence, often far away from the primary tumor site [15]. Moreover, integrin-ECM interactions have been shown to increase cell survival after radiation exposure [13]. Supplementary to their role in cancer cells, integrins on the surface of host cells (e.g., endothelial cells, perivascular cells, fibroblasts etc.) in the neoplastic microenvironment can boost the malignant potential of a tumor by mediating angiogenesis, lymphangiogenesis, and desmoplasia [16]. These facts make integrins an attractive target for anti-tumor therapy, the potential effects being on curtailing angiogenesis, invasion, and tumor growth, see e.g., [16, 62]. Among the types of integrin inhibitors evaluated in preclinical or clinical studies, peptidomimetics $\left(\mathrm{RGD}^{3}\right.$-based small protein-like chains designed to mimic peptides and blocking ligand binding) are aimed at treating glioblastoma [11]. Examples of pseudomimetics are cilengitide (targets $\alpha_{v} \beta_{3} \mid \alpha_{v} \beta_{6}$ integrins), ATN 161 (targets $\alpha_{5} \beta_{1}$ integrins), and HYD1 (targets $\beta_{1}$ integrins) [11]. In our model we will consider the action of such chemotherapeutic agents.

The effects of chemotherapy are described in our equations by way of dependence on the chemotherapeutic dose $d_{c}$. Hence the influences are on the transition rates between proliferating and migrating phenotypes, on the growth function of the (resting) tumor cells, and on the binding of free receptors to the tissue fraction surviving irradiation (attachment and detachment rates $k^{+}\left(d_{c}\right)$ and $k^{-}\left(d_{c}\right)$, respectively, in (4)).

The microscale dynamics of receptor binding is characterized by

$$
\dot{y}=G\left(y, Q, d_{c}, d_{r}\right)=k^{+}\left(d_{c}\right)(1-y) Q S\left(\alpha_{3}, d_{r}\right)-k^{-}\left(d_{c}\right) y .
$$

In equations (1) and (2) above, the function $N$ denotes the total glioma cell density and is given by

$$
N(t, \mathbf{x})=\int_{V} \int_{Y} p(t, \mathbf{x}, \mathbf{v}, y) d y d \mathbf{v}+\int_{Y} r(t, \mathbf{x}, y) d y .
$$

\subsection{Derivation of the effective equations on the macroscopic level}

The model in Subsection 2.1 is a system coupling partial differential equations with an ordinary differential equation. Its well posedness will be addressed in Subsection 2.3, along with that of the

\footnotetext{
${ }^{2}$ The standard treatment for newly diagnosed glioblastoma consists of maximal surgical resection, radiotherapy, and concomitant and adjuvant chemotherapy with temozolomide, see e.g., [56, 22].

${ }^{3}$ arginylglycylaspartic acid
} 
macroscale equation for $N$ obtained in this subsection via parabolic scaling. Before doing this scaling, however, we normalize the subcellular dynamics as in $[17,19,18]$. The simplified equation becomes

$$
\dot{y}=k^{+}\left(d_{c}\right)(1-y) Q S\left(\alpha_{3}, d_{r}\right)-k^{-}\left(d_{c}\right) y=-\left(k^{+} Q S+k^{-}\right) y+k^{+} Q S .
$$

The unique steady state of this equation is given by

$$
y^{*}=\frac{k^{+} Q S}{k^{+} Q S+k^{-}}=f .
$$

As in [19] we introduce the deviation $z:=f-y$ from the steady state and consider the path of a single cell starting in $\mathbf{x}_{0}$ and moving with velocity $\mathbf{v}$ through a time-invariant density field $Q(\mathbf{x})$. Then with the notation $\mathbf{x}=\mathbf{x}_{0}+\mathbf{v} t$ it follows that $z$ satisfies the equation

$$
\dot{z}=\dot{f}-\dot{y}=-\left(k^{+} Q S+k^{-}\right) z+F(t)+f^{\prime}(Q) \mathbf{v} \cdot \nabla Q(\mathbf{x}),
$$

where

$$
F(t):=\dot{d}_{c}\left(\frac{\partial f}{\partial k^{+}}\left(k^{+}\right)^{\prime}\left(d_{c}\right)+\frac{\partial f}{\partial k^{-}}\left(k^{-}\right)^{\prime}\left(d_{c}\right)\right)+\dot{d}_{r} f^{\prime}(S) S^{\prime}\left(\alpha_{3}, d_{r}\right) .
$$

Thus, choosing (as in $[17,19]$ the turning rate to be of the form $\lambda(z)=\lambda_{0}-\lambda_{1} z \geq 0$, where $\lambda_{0}$ and $\lambda_{1}$ are some positive constants, the transformed system of equations reads:

$$
\begin{aligned}
\partial_{t} p+\nabla \cdot(\mathbf{v} p) & -\frac{\partial}{\partial z}\left(\left(\left(k^{+} Q S+k^{-}\right) z-F(t)-f^{\prime}(Q) \mathbf{v} \cdot \nabla Q\right) p\right) \\
& =-\lambda_{0} p+\lambda_{0} \frac{q(\hat{\mathbf{v}})}{\omega} \bar{p}+\lambda_{1} z p-\lambda_{1} z \frac{q(\hat{\mathbf{v}})}{\omega} \bar{p}-a\left(\mathbf{x}, d_{c}\right) p+b\left(\mathbf{x}, d_{c}\right) \frac{q(\hat{\mathbf{v}})}{\omega} r-L_{1}\left(N, \alpha_{1}, d_{r}\right) p \\
\partial_{t} r & =a\left(\mathbf{x}, d_{c}\right) \bar{p}-b\left(\mathbf{x}, d_{c}\right) r+g\left(N, d_{c}\right) r-L_{2}\left(N, \alpha_{2}, d_{r}\right) r
\end{aligned}
$$

where $\bar{p}(t, \mathbf{x}, y):=\int_{V} p(\mathbf{v}) d \mathbf{v}$ and $L_{i}\left(N, \alpha_{i}, d_{r}\right):=l_{i}(N)+R_{i}\left(\alpha_{i}, d_{r}\right), i=1,2$.

Now doing the parabolic scaling $x \rightarrow \epsilon x, t \rightarrow \epsilon^{2} t$ we obtain

$$
\begin{aligned}
\epsilon^{2} \partial_{t} p+\epsilon \nabla \cdot(\mathbf{v} p) & -\frac{\partial}{\partial z}\left(\left(\left(k^{+} Q S+k^{-}\right) z-\epsilon^{2} F(t)-\epsilon f^{\prime}(Q) \mathbf{v} \cdot \nabla Q\right) p\right) \\
& =-\lambda_{0} p+\lambda_{0} q \bar{p}+\lambda_{1} z p-\lambda_{1} z \frac{q}{\omega} \bar{p}-a\left(d_{c}\right) p+b\left(d_{c}\right) \frac{q}{\omega} r-\epsilon^{2} L_{1}\left(N, \alpha_{1}, d_{r}\right) p \\
\epsilon^{2} \partial_{t} r & =a\left(d_{c}\right) \bar{p}-b\left(d_{c}\right) r+\epsilon^{2} g\left(N, d_{c}\right) r-\epsilon^{2} L_{2}\left(N, \alpha_{2}, d_{r}\right) r .
\end{aligned}
$$

Thereby, we scaled with $\epsilon^{2}$ the quantity $F(t)$ involving time derivatives of the different doses and the survival fraction $S$ and accounting for fast dynamics.

In the next step we set up a moment system w.r.t. the involved distribution functions and introduce the notations

$$
\begin{array}{rlrl}
m(t, \mathbf{x}, \mathbf{v}) & =\int_{Z} p(t, \mathbf{x}, \mathbf{v}, z) d z & M(t, \mathbf{x}) & =\int_{V} m(t, \mathbf{x}, \mathbf{v}) d \mathbf{v} \\
m^{z}(t, \mathbf{x}, \mathbf{v}) & =\int_{Z} z p(t, \mathbf{x}, \mathbf{v}, z) d z & M^{z}(t, \mathbf{x}) & =\int_{V} m^{z}(t, \mathbf{x}, \mathbf{v}) d \mathbf{v} \\
W(t, \mathbf{x}) & =\int_{Z} r(t, \mathbf{x}, z) d z & W^{z}(t, \mathbf{x})=\int_{Z} z r(t, \mathbf{x}, z) d z
\end{array}
$$

where $Z \subseteq\left[y^{*}-1, y^{*}\right]$ is our new domain for the internal dynamics. The higher order moments are neglected, in virtue of the subcellular dynamics being much faster than the events on the higher scales, which permits to assume $z$ to be close to zero (i.e., the steady state of the subcellular dynamics is rapidly reached). As in $[17,19,18]$ we assume the functions to have a relatively compact support in this interval and be compactly supported in the $(\mathbf{x}, \mathbf{v})$-space, which allows to perform the subsequent 
calculations.

With the moment notations we get (after integrating (10) and (11) w.r.t. $z$ ):

$$
\begin{aligned}
\epsilon^{2} \partial_{t} m+\epsilon \mathbf{v} \cdot \nabla m= & -\lambda_{0} m+\lambda_{0} \frac{q}{\omega} M+\lambda_{1} m^{z}-\lambda_{1} \frac{q}{\omega} M^{z}-a m+b \frac{q}{\omega} W-\epsilon^{2} L_{1} m \\
\epsilon^{2} \partial_{t} W= & a M-b W+\epsilon^{2}\left(g-L_{2}\right) W \\
\epsilon^{2} \partial_{t} m^{z}+\epsilon \mathbf{v} \cdot \nabla m^{z}= & -\left(k^{+} Q S+k^{-}\right) m^{z}+\epsilon f^{\prime}(Q) \mathbf{v} \cdot \nabla Q m+\epsilon^{2} F m \\
& -\lambda_{0} m^{z}+\lambda_{0} \frac{q}{\omega} M^{z}-a m^{z}+b \frac{q}{\omega} W^{z}-\epsilon^{2} L_{1} m^{z} \\
\epsilon^{2} \partial_{t} W^{z}= & a M^{z}-b W^{z}+\epsilon^{2}\left(g-L_{2}\right) W^{z}
\end{aligned}
$$

Now we consider Hilbert expansions $\Xi=\sum_{k=0}^{\infty} \Xi_{k} \epsilon^{k}$, for $\Xi \in\left\{m, m^{z}, M, M^{z}, W, W^{z}\right\}$ and collect corresponding powers of $\epsilon$ :

$\epsilon^{0}:$

$$
\begin{aligned}
& 0=-\left(\lambda_{0}+a\right) m_{0}+\lambda_{0} \frac{q}{\omega} M_{0}+\lambda_{1} m_{0}^{z}-\lambda_{1} \frac{q}{\omega} M_{0}^{z}+b \frac{q}{\omega} W_{0} \\
& 0=a M_{0}-b W_{0} \\
& 0=-\left(k^{+} Q S+k^{-}+\lambda_{0}+a\right) m_{0}^{z}+\lambda_{0} \frac{q}{\omega} M_{0}^{z}+b \frac{q}{\omega} W_{0}^{z} \\
& 0=a M_{0}^{z}-b W_{0}^{z} .
\end{aligned}
$$

From these equations we deduce by integrating w.r.t. $\mathbf{v}$ (where appropriate) that $M_{0}^{z}=0, W_{0}^{z}=0$, $m_{0}^{z}=0, m_{0}=\frac{q}{\omega} M_{0}$, and $W_{0}=\frac{a}{b} M_{0}$.

$\epsilon^{1}$ :

$$
\begin{aligned}
\mathbf{v} \cdot \nabla m_{0} & =-\left(\lambda_{0}+a\right) m_{1}+\lambda_{0} \frac{q}{\omega} M_{1}+\lambda_{1} m_{1}^{z}-\lambda_{1} \frac{q}{\omega} M_{1}^{z}+b \frac{q}{\omega} W_{1} \\
0 & =a M_{1}-b W_{1} \\
\mathbf{v} \cdot \nabla m_{0}^{z} & =-\left(k^{+} Q S+k^{-}+\lambda_{0}+a\right) m_{1}^{z}+f^{\prime}(Q) \mathbf{v} \cdot \nabla Q m_{0}+\lambda_{0} \frac{q}{\omega} M_{1}^{z}+b \frac{q}{\omega} W_{1}^{z} \\
0 & =a M_{1}^{z}-b W_{1}^{z} .
\end{aligned}
$$

Using the above deduced facts in (19), then in (18) (integrated w.r.t. v) and (16) we obtain $M_{1}^{z}=$ $W_{1}^{z}=0$

$$
\begin{aligned}
& m_{1}^{z}=\frac{1}{\gamma(\mathbf{x})} f^{\prime}(Q) \mathbf{v} \cdot \nabla Q m_{0} \\
& m_{1}=\frac{1}{\lambda_{0}+a}\left(\frac{\lambda_{1}}{\gamma} f^{\prime}(Q) \mathbf{v} \cdot \nabla Q m_{0}-\mathbf{v} \cdot \nabla m_{0}+\frac{a q}{\omega} M_{1}\right)
\end{aligned}
$$

where $\gamma(\mathbf{x}):=k^{+} Q S+k^{-}+\lambda_{0}+a$.

By (16) and (17) we can write

$$
\mathbf{v} \cdot \nabla m_{0}=\mathcal{L}\left[\lambda_{0}+a\right] m_{1}+\lambda_{1} m_{1}^{z} .
$$

Then $\mathcal{L}\left[\lambda_{0}+a\right]$ defined on the weighted $L^{2}$-space $L_{q}^{2}(V)$ with the weight function $q^{-1}(\hat{\mathbf{v}})$ is a compact Hilbert-Schmidt operator (see [30]) with pseudoinverse $\mathcal{L}\left[\lambda_{0}+\alpha\right]_{\mid<q>\perp}^{-1} \zeta=-\frac{1}{\lambda_{0}+\alpha} \zeta$. As in [19], this leads to

$$
m_{1}=-\frac{1}{\lambda_{0}+\alpha}\left(\mathbf{v} \cdot \nabla m_{0}-\lambda_{1} m_{1}^{z}\right) .
$$

We also have the set of equations $\epsilon^{2}:$

$$
\begin{aligned}
\partial_{t} m_{0}+\mathbf{v} \cdot \nabla m_{1} & =\mathcal{L}\left[\lambda_{0}\right] m_{2}+\lambda_{1} m_{2}^{z}-\lambda_{1} \frac{q}{\omega} M_{2}^{z}-a m_{2}+b \frac{q}{\omega} W_{2}-L_{1}\left(N_{0}\right) m_{0} \\
\partial_{t} W_{0} & =a M_{2}-b W_{2}+\left(g\left(N_{0}\right)-L_{2}\left(N_{0}\right)\right) W_{0},
\end{aligned}
$$


From (24) we have

$$
\frac{b}{\omega} W_{2}=\frac{1}{\omega}\left[a M_{2}+\left(g\left(N_{0}\right)-L_{2}\left(N_{0}\right)\right) W_{0}-\partial_{t} W_{0}\right]
$$

which plugged into (23) leads to

$$
\partial_{t} m_{0}+\mathbf{v} \cdot \nabla m_{1}=\mathcal{L}\left[\lambda_{0}+a\right] m_{2}-\mathcal{L}\left[\lambda_{1}\right] m_{2}^{z}+\frac{q}{\omega}\left[\frac{a}{b} M_{0}\left(g\left(N_{0}\right)-L_{2}\left(N_{0}\right)\right)-\partial_{t}\left(W_{0}\right)\right]-L_{1}\left(N_{0}\right) m_{0} .
$$

After integrating w.r.t. $\mathbf{v}$ and rearranging this becomes

$$
\partial_{t} M_{0}+\int_{V} \mathbf{v} \cdot \nabla m_{1} d \mathbf{v}=\left(g\left(N_{0}\right)-L_{2}\left(N_{0}\right)\right) W_{0}-\partial_{t} W_{0}-L_{1}\left(N_{0}\right) M_{0} .
$$

Hence with $N_{0}=M_{0}+W_{0}=\left(1+\frac{a}{b}\right) M_{0}$ we get

$$
\begin{aligned}
\partial_{t} N_{0}+\nabla \cdot \int_{V}\left(\mathbf{v} m_{1}\right) d \mathbf{v} & =\left(g\left(N_{0}\right)-L_{2}\left(N_{0}\right)\right) W_{0}-L_{1}\left(N_{0}\right) M_{0} \\
& =\frac{a}{a+b} N_{0}\left(g\left(N_{0}\right)-L_{2}\left(N_{0}\right)\right)-\frac{b}{a+b} N_{0} L_{1}\left(N_{0}\right) .
\end{aligned}
$$

It remains to express $\int_{V} \mathbf{v} m_{1} d \mathbf{v}$ in terms of $N_{0}$. From (22) we have

$$
m_{1}=-\frac{1}{\lambda_{0}+\alpha}\left(\mathbf{v} \cdot \nabla\left(\frac{q}{\omega} M_{0}\right)-\lambda_{1} m_{1}^{z}\right)
$$

and from (20) we know $m_{1}^{z}=\frac{1}{\gamma(\mathbf{x})} f^{\prime}(Q) \mathbf{v} \cdot \nabla Q \frac{q}{\omega} M_{0}$, hence

$\nabla \cdot \int_{V} \mathbf{v} m_{1} d \mathbf{v}=\nabla \cdot\left(-\frac{1}{\lambda_{0}+a} \nabla \cdot\left(\frac{1}{\omega} \int_{V} \mathbf{v v}^{t} q d \mathbf{v} M_{0}\right)\right)+\nabla \cdot\left(\frac{\lambda_{1}}{\lambda_{0}+a} \frac{1}{\gamma(\mathbf{x})} f^{\prime}(Q) \frac{1}{\omega} \int_{V} \mathbf{v v}^{t} q d \mathbf{v} \nabla Q M_{0}\right)$.

Now denote

$$
\mathbb{D}_{T}(\mathbf{x}):=\frac{1}{\omega} \int_{V} \mathbf{v v}^{t} q d \mathbf{v}
$$

to obtain

$$
\nabla \cdot \int_{V} \mathbf{v} m_{1} d \mathbf{v}=\nabla \cdot\left(-\frac{1}{\lambda_{0}+a} \nabla \cdot\left(\frac{b}{a+b} \mathbb{D}_{T}(\mathbf{x}) N_{0}\right)\right)+\nabla \cdot\left(\frac{\lambda_{1}}{\lambda_{0}+a} \frac{1}{\gamma(\mathbf{x})} \frac{b}{a+b} f^{\prime}(Q) \mathbb{D}_{T}(\mathbf{x}) \nabla Q N_{0}\right) .
$$

Plug this into (25) to obtain the macroscopic equation for $N_{0}$ :

$$
\begin{aligned}
\partial_{t} N_{0}-\nabla \cdot\left(\frac{1}{\lambda_{0}+a} \nabla \cdot\left(\frac{b}{a+b} \mathbb{D}_{T}(\mathbf{x}) N_{0}\right)\right. & )+\nabla \cdot\left(\frac{\lambda_{1} f^{\prime}(Q)}{\gamma(\mathbf{x})} \frac{b}{\left(\lambda_{0}+a\right)(b+a)} \mathbb{D}_{T}(\mathbf{x}) \nabla Q N_{0}\right) \\
= & \left(\left(g\left(N_{0}\right)-L_{2}\left(N_{0}\right)\right) \frac{a}{a+b}-L_{1}\left(N_{0}\right) \frac{b}{a+b}\right) N_{0}
\end{aligned}
$$

Throughout the rest of this paper we will assume for simplicity that the functions $a$ and $b$ depend only on time.

\subsection{Well posedness of the settings}

\subsubsection{Micro-meso system}

The system (1), (2), (4) fits in the more general framework handled in [41], hence its well posedness follows (with corresponding initial conditions) like in that reference. 


\subsubsection{Macroscopic equation}

For the well posedness of the macroscopic equation (26) set in a bounded space-time domain we rely on the theory of monotone operators for nonlinear parabolic equations (see e.g. [53]).

Let $\Omega$ be bounded domain in $\mathbb{R}^{3}$ with Lipschitz boundary $\partial \Omega$. We want to verify the existence of a solution to the nonlinear parabolic initial-boundary-value problem

$$
\begin{aligned}
\partial_{t} u-\nabla \cdot(D(\mathbf{x}, t) \nabla u-H(\mathbf{x}, t) u)+G(u) & =0 & & \text { in } \Omega \times(0, T) \\
(D(\mathbf{x}, t) \nabla u-H(\mathbf{x}, t) u) \cdot \mathbf{n} & =0 & & \text { on } \partial \Omega \times(0, T) \\
u(0) & =u_{0} & & \text { on } \Omega \times\{0\},
\end{aligned}
$$

where

$$
\begin{aligned}
D(\mathbf{x}, t) & =\frac{b(t)}{\left(\lambda_{0}+a(t)\right)(a(t)+b(t))} \mathbb{D}_{T}(\mathbf{x}) \\
H(\mathbf{x}, t) & =\frac{\lambda_{1} f^{\prime}(Q)}{\gamma(t, \mathbf{x})} \frac{b(t)}{\left(\lambda_{0}+a(t)\right)(b(t)+a(t))} \mathbb{D}_{T}(\mathbf{x}) \nabla Q-\frac{b(t)}{\left(\lambda_{0}+a(t)\right)(a(t)+b(t))} \operatorname{div}\left(\mathbb{D}_{T}(\mathbf{x})\right) \\
G(u, t) & =-\left(\left(g(u, t)-L_{2}(u, t)\right) \frac{a(t)}{a(t)+b(t)}-L_{1}(u, t) \frac{b(t)}{a(t)+b(t)}\right) u .
\end{aligned}
$$

\section{Assumptions:}

(A.i) The functions $D$ and $H$ are continuous in time and essentially bounded in space. Moreover, $D$ satisfies the condition $\xi^{T} D(\mathbf{x}, t) \xi \geq \theta(t)|\xi|^{2}$ with a positive $\theta(t)$ bounded away from zero: $0<c_{\theta} \leq \theta(t) \leq C_{\theta}<\infty$ for all $t \geq 0$.

(A.ii) $G$ is continuous w.r.t. time and the solution variable. Moreover, $G$ satisfies the condition $G(0, t)=0$, the growth condition $|G(u, t)| \leq C\left(1+|u|^{2}\right)$, with a constant $C$ independent of time and space, and the coercivity condition $\inf _{\zeta \in \mathbb{R}^{+}} G(\zeta, t) \zeta>-\infty$.

For our concrete setting above we require that:

(i) The diffusion tensor $\mathbb{D}_{T}$ is uniformly positive definite and lies in the space $W^{1, \infty}(\Omega)$.

(ii) The volume fraction of tissue fibers $Q$ has to lie in the space $W^{1, \infty}(\Omega)$, as well.

(iii) The rates $a, b, k^{+}$and $k^{-}$are continuous in the variable $d_{c}$ (which has to be continuous in time), and uniformly bounded.

(iv) The gain and loss functions $g$ and $l$ (the latter is contained in the expression of $L_{i}, i=1,2$ ) are continuous and bounded.

Remark 2.1. Assumptions (i) and (ii) express the fact that the intrinsic properties of the brain structure are smooth. This can be justified at a reasonable level of detail. Moreover, the diffusion tensor $\mathbb{D}_{T}$ is (re)constructed in such a way that the uniform positive definiteness is assured in every computational voxel.

Lemma 2.1. For non-negative initial data $u_{0}$ a solution of (27) remains non-negative for all future times.

Proof. This follows immediately from our assumptions, by applying the parabolic comparison principle.

As we are only interested in non-negative initial values, leading to non-negative solutions, we assume in the following that $G(t, u)=0$ for $u<0$.

Define the spaces $V:=H^{1}(\Omega)$ and $H:=L^{2}(\Omega)$ and the corresponding Gelfand triple $\left(V, H, V^{\star}\right)$. We look for a solution to $(27)$ in the space $W:=\left\{v \in L^{2}(0, T ; V), v^{\prime} \in L^{2}\left(0, T ; V^{\star}\right)\right\}$, with $T>0$ fixed. 
Now define the operators

$$
\begin{aligned}
\langle A(t) u, v\rangle & :=\int_{\Omega}(D(\mathbf{x}, t) \nabla u-H(\mathbf{x}, t) u) \cdot \nabla v d \mathbf{x} \\
\langle B(t) u, v\rangle & :=\int_{\Omega} G(u, t) v d \mathbf{x}
\end{aligned}
$$

for $u, v \in W$.

Remark 2.2. The operators $A$ and $B$ defined above are continuous w.r.t. time, due to (iii).

Lemma 2.2. The family of operators $A(t)$ is continuous from $V$ to $V^{\star}$ and satisfies Garding's inequality

$$
\langle A(t) u, u\rangle \geq c_{1}\|u\|_{V}^{2}-c_{2}\|u\|_{H}^{2}
$$

with the constants $c_{1}>0$ and $c_{2} \geq 0$.

Proof. The claim follows straightforwardly by usual estimates.

Remark 2.3. We assume without loss of generality that $c_{2}=0$. Otherwise we transform (27) into an equivalent problem by $\hat{u}=\exp \left(c_{2} t\right) u$. Note that after this transformation the operators $A$ and $B$ remain continuous in time.

The following result is obtained (as a particular case) by a simple adaptation of the proofs in Section 3.3.6, [53] (or of section III.4, [58]):

Theorem 2.1. Let $u_{0} \in H$. Under the previous assumptions about the function $G$ and the coefficients involved in $A$ there exists a solution $u \in W$ to (27), in the sense that

$$
-\int_{0}^{T}\left\langle v^{\prime}(t), u(t)\right\rangle_{V} d t+\int_{0}^{T}\langle A(t) u(t), v(t)\rangle_{V} d t+\int_{0}^{T}\langle B(t) u(t), v(t)\rangle_{V}=\left(u_{0}, v(0)\right)_{H}
$$

for all $v \in W$ with $v(T)=0$. If $G$ is strictly monotone, then the solution $u$ is unique.

\section{Assessing the parameters and coefficient functions}

\subsection{Fiber density $q$}

To determine the diffusion tensor $\mathbb{D}_{T}$ we need to determine the fiber orientation distribution $q$. As is [17, 19, 18] we could select $q$ to be the peanut distribution proposed by Hillen \& Painter in [31]:

$$
q(\mathbf{x}, \boldsymbol{\theta})=\frac{n}{\left|\mathbb{S}^{n-1}\right| \operatorname{tr}\left(\mathbb{D}_{W}(\mathbf{x})\right)} \boldsymbol{\theta}^{t} \mathbb{D}_{W} \boldsymbol{\theta},
$$

where $\mathbb{D}_{W}$ denotes the DTI-measured water diffusion tensor and $\boldsymbol{\theta} \in \mathbb{S}^{n-1}$ gives the fiber orientation. The major problem occurring with this choice is that the water diffusion tensor may not reproduce the brain structure in enough detail, as it consists of only six gradient directions and cannot resolve crossing fiber tracts [8]. Different other choices try to overcome this drawback. One is to use a bimodal von Mises-Fisher distribution (see [43]) as proposed in [47]

$$
q(\mathbf{x}, \boldsymbol{\theta})=\frac{k(\mathbf{x})}{8 \pi \sinh (k(\mathbf{x}))}(\exp (k(\mathbf{x}) \phi \cdot \boldsymbol{\theta})+\exp (-k(\mathbf{x}) \phi \cdot \boldsymbol{\theta})),
$$

where $k(\mathbf{x})=\kappa F A(\mathbf{x})$, with the measured fractional anisotropy $F A$ and a real constant $\kappa$ to be determined. The vector $\phi$ represents the leading eigenvector of the diffusion tensor for each voxel. This choice, however, has several disadvantages: On the one hand, the parameter $\kappa$ cannot be measured and has to be assessed by using different clinical DTI data sets. On the other hand, the fractional anisotropy is a not satisfactory enough indicator for anisotropy (see [66]), as well as for the fiber density. Moreover, the leading eigenvector does not resemble the fiber orientation in all voxels [34]. Another way to enhance the quality of the diffusion tensor description is to use the concept of an orientation 
distribution function $(\mathrm{ODF})[8,1]$ which describes the probability of diffusion in a direction $\boldsymbol{\theta}$. The usual definition of this probability is

$$
O D F(\boldsymbol{\theta})=\int_{0}^{\infty} \mathcal{P}(r \boldsymbol{\theta}) r^{2} d r,
$$

where $\mathcal{P}(r \boldsymbol{\theta})$ denotes the displacement probability of a spatial point in spherical coordinates. ${ }^{4}$ For more details we refer to the cited paper. Hence we may set our fiber orientation density to the orientation distribution function

$$
q(\boldsymbol{\theta})=O D F(\boldsymbol{\theta}) .
$$

For diffusion tensor imaging the fiber orientation $q$ in (30) can be computed explicitly ([1]):

$$
q(\mathbf{x}, \boldsymbol{\theta})=\frac{1}{4 \pi\left|\mathbb{D}_{W}(\mathbf{x})\right|\left(\boldsymbol{\theta}^{t} \mathbb{D}_{W}(\mathbf{x})^{-1} \boldsymbol{\theta}\right)^{\frac{3}{2}}},
$$

thus we do not need reconstruction procedures involving the integral expression to obtain this quantity. The ODF is available for different medical imaging techniques [1, 8], among them also the high quality imaging procedures Q-Ball and HARDI. This is a major advantage allowing to include different medical data in the model. For our test data ${ }^{5}$ we use here this ODF; the issue of comparison between this and the peanut distribution for the tumor diffusion tensor setup will be addressed in a forthcoming work.

\subsection{Estimation of $Q$}

We estimated the volume fraction of tissue fibers $Q$ as in [18]. Hence we chose

$$
Q=1-\frac{l_{c}^{3}}{h^{3}}
$$

where $h$ is the side length of one voxel and the characteristic length $l_{c}$ is estimated via

$$
l_{c}=\sqrt{\frac{\operatorname{tr}\left(\mathbb{D}_{W}\right) h^{2}}{4 l_{1}}},
$$

using $l_{1}$ as the leading eigenvalue of the diffusion tensor $\mathbb{D}_{W}$. The determination of an unbiased estimator for the fiber volume fraction is ongoing work.

\subsection{Coefficient functions}

First we have to select the transition rates $a$ and $b$ between the migrating population $p$ in (1) and the proliferating population $r$ in (2). These functions depend on the space variable and the dose of chemotherapeutic agent, however as mentioned before we choose them constant w.r.t. $\mathbf{x}$, as there is no known procedure to quantify this dependency. As mentioned in Section 2, the chemotherapeutic drug modeled in this paper concentrates its impact on the integrin bindings. These are essential for cell migration, thus this has to be taken into account for the choice of $a$ and $b$. The rate $a$ models the cell transition from the migrating to the resting (and hence proliferating) regime. As migrating cells seem to be responsible for the infiltrative behavior of the tumor and its recurrence, we aim at inhibiting the migratory phenotype of the cell population. Hence, $a$ should be monotonously increasing in its only variable $d_{c}$. The rate $b$ describes the transition rate to migratory behavior, so it has to be monotonically decreasing in $d_{c}$. Therefore, a possible choice is

$$
\begin{aligned}
& a\left(d_{c}\right)=0.05 \cdot\left(1+d_{c}\right) \\
& b\left(d_{c}\right)=0.1 \cdot\left(1+\frac{d_{c}}{1+d_{c}^{2}}\right) .
\end{aligned}
$$

\footnotetext{
${ }^{4}$ Note that the factor $r^{2}$ in this expression is often left out, but [1] argued that it is actually essential when considering normalized solid angles.

${ }^{5}$ provided by Carsten Wolters, Institute of Biosignal Analysis, WWU Münster, see [64]
} 
As there is no quantitative information available for these rates, they cannot be fit to measurements. Therefore, we concentrate on their qualitative behavior. This also applies to the rates $k^{+}$and $k^{-}$. However, there is some indication that this choice is reasonable, because it is of the same order as the constants $\alpha$ and $\beta$ [19] modeling the same rates. The unit of these rates is $\frac{1}{\mathrm{~s}}$.

Next we address the receptor binding rates $k^{+}$and $k^{-}$, the first of which describes the rate of a cell binding to unsoluble ligands (fibers) in its environment, while $k^{-}$is the detachment rate. Since the chemotherapeutical agent under consideration is meant to inhibit receptor bindings, we assume the function $k^{+}$to be monotonically decreasing, while $k^{-}$is supposed to be monotonically increasing. This means that it is more likely for a cell under the influence of the chemotherapeutical substance to detach from the ECM than to attach to it. Thus our choices are

$$
\begin{aligned}
& k^{+}\left(d_{c}\right)=0.1 \cdot\left(1+\frac{d_{c}}{1+d_{c}^{2}}\right) \\
& k^{-}\left(d_{c}\right)=0.1 \cdot\left(1+d_{c}\right) .
\end{aligned}
$$

This corresponds to the rates selected in $[19,18]$. The rate $k^{-}$could be determined to be around 0.1 in absence of the chemotherapeutical substance [38]. The rate $k^{+}$should be larger than $k^{-}$, since the attachment of the cell to the surrounding fibers should be more probable then detachment, if no further (bio)chemical information is available. Like for the rates $a$ and $b$, the unit of $k^{+}$and $k^{-}$is $\frac{1}{\mathrm{~s}}$. Eventually we have to adjust the functions included in the nonlinear term $G$ and describing radiotherapy and proliferation. Like in [19, 18], we model the growth in a logistic way and select

$$
\begin{gathered}
g(s)=c_{g} \\
L_{1}\left(d_{c}, d_{r}, u\right)=c_{g} u+\sum_{i=1}^{\nu}\left(1-S\left(\alpha_{1}, d_{r}\right)\right) \eta_{\delta}\left(t-t_{i}\right) \\
L_{2}\left(d_{c}, d_{r}, u\right)=c_{g} u+\sum_{i=1}^{\nu}\left(1-S\left(\alpha_{2}, d_{r}\right)\right) \eta_{\delta}\left(t-t_{i}\right) .
\end{gathered}
$$

As in (3), $\nu$ is the number of therapy fractions. Altogether we have

$-G(w)=\frac{a}{a+b} c_{g} w-c_{g} w^{2}-\left(\frac{b}{a+b} \sum_{i=1}^{\nu}\left(1-S\left(\alpha_{1}, d_{r}\right)\right) \eta_{\delta}\left(t-t_{i}\right)+\frac{a}{a+b} \sum_{i=1}^{\nu}\left(1-S\left(\alpha_{2}, d_{r}\right)\right) \eta_{\delta}\left(t-t_{i}\right)\right) w$.

Note that this $G$ fulfills the requirements of Assumption $(A . i i)$. The growth rate $c_{g}$ has to be measured in an appropriate way. In [18] we used information about the cell cycle to deduce $c_{g}$ to be approximately $8 \cdot 10^{-7} \frac{1}{\mathrm{~s}}-10^{-6} \frac{1}{\mathrm{~s}}$.

\subsection{Constants}

We selected the necessary constants as in Table 1 below. The necessary therapy parameters change from scenario to scenario, so we will specify them where occurring.

\section{Numerical simulations}

We solve the equation (26). While all necessary data, i.e. the diffusion tensor $\mathbb{D}_{T}$ and the volume fraction of tissue fibers $Q$, are computed in advance from the DTI measurements using $\mathrm{C}++$ and the Armadillo linear algebra library [55], we implemented the simulation of the PDE via the numerical framework DUNE [7, 5, 4, 3]. The coefficients and the drift term depend on time and space, so we expect time dependent regions of the computational domain that are dominated by the diffusion term and others dominated by the drift term. Thus we need numerical methods capable to handle both diffusion dominated and degenerate parabolic equations. Moreover, the selected method has to handle full tensors and it should be locally mass conservative and positivity preserving. 


\begin{tabular}{|c|c|l|}
\hline Parameter & Value & Source \\
\hline$s$ & $0.21 \cdot 10^{-6} \frac{m}{\mathrm{~s}}$ & {$[12]$} \\
\hline$\lambda_{0}$ & $0.8 \frac{1}{\mathrm{~s}}$ & K. Wolf (Radboud Univ. Nijmegen), unpublished data \\
\hline$\lambda_{1}$ & $10000 \frac{1}{\mathrm{~s}}$ & estimated, corresponds to [17] \\
\hline$c_{g}$ & $\approx 8 \cdot 10^{-7} \frac{1}{\mathrm{~s}}-10^{-6} \frac{1}{\mathrm{~s}}$ & chosen as in [18]. \\
\hline$\alpha_{1}$ & $\frac{0.033}{\mathrm{~Gy}}-\frac{0.058}{\mathrm{~Gy}}$ & corresponding to the glioma cell line TK1 in [6] \\
\hline$\alpha_{2}$ & $\frac{0.311}{\mathrm{~Gy}}-\frac{0.401}{\mathrm{~Gy}}$ & corresponding to the glioma cell line A172 in [6] \\
\hline$\alpha_{3}$ & $\frac{0.37}{\mathrm{~Gy}}$ & corresponding to fibroblasts in [6] \\
\hline$\beta_{1}$ & $\frac{0.047}{\mathrm{~Gy}^{2}}-\frac{0.048}{\mathrm{~Gy}^{2}}$ & corresponding to the glioma cell line TK1 in [6] \\
\hline$\beta_{2}$ & $\frac{0.061}{\mathrm{~Gy}^{2}}-\frac{0.091}{\mathrm{~Gy}^{2}}$ & corresponding to the glioma cell line A172 in [6] \\
\hline$\beta_{3}$ & $\frac{0.016}{\mathrm{~Gy}^{2}}-\frac{0.052}{\mathrm{~Gy}^{2}}$ & corresponding to fibroblasts in [6] \\
\hline
\end{tabular}

Table 1: Model parameters

\subsection{Implementation}

For the simulations we use a parallel structured quadrilateral mesh as implemented in YaspGrid of DUNE. The cells are chosen in such a way that we have a subset of the voxel mesh given by the medical data set consisting of the regions occupied by gray and white matter. These were given by a segmentation of the brain in the data set. On this mesh we use a cell centered finite volume method as described in [20]. For the time discretization we employ an implicit Euler scheme with a step size $\tau$ satisfying a CFL-condition near 1 . In our case, we considered $\tau$ to be one half of a day.

\subsection{Results}

We performed numerical simulations for different scenarios. The coefficients are those given in Section 3. Different therapy strategies are to be compared, all of which involve resection followed after 21 days ${ }^{6}$ by radio- and chemotherapy, the latter applied in a concurrent way. The starting point is considered the detection of the tumor. Recall that here the chemotherapy is aiming merely at inhibiting receptor binding to the tissue fibers; the cell kill is achieved by radiotherapy.

Strategy 1: Resection (two weeks after start), no further therapy.

Strategy 2: $\quad$ Resection (two weeks after start), followed after three weeks by radiotherapy (a daily dose of 2 Gy - except on weekends) for six weeks

Strategy 3: $\quad$ Resection (two weeks after start), followed after three weeks by concurrent chemotherapy (a normalized dose of 5.0 in our model) and radiotherapy (a daily dose of 2 Gy - except on weekends) for six weeks.

As therapies with inhibitors of receptor/integrin binding to unsoluble ligands in the environment of tumor cells are not yet approved for clinical practice, we relied on some clinical trials when designing the strategies 1 to 4 , thereby intentionally omitting the effect of a chemotherapeutic agent (like temozolomide) directly aiming at cell kill. Hence, these strategies (and the involved parameters) are motivated by the trials NCT01165333 (Cilengitide in Combination With Irradiation in Children With Diffuse Intrinsic Pontine Glioma) and NCT00689221 (Cilengitide, Temozolomide, and Radiation Therapy in Treating Patients With Newly Diagnosed Glioblastoma and Methylated Gene Promoter Status) at ClinicalTrials.gov.

In our simulations the resection is numerically obtained by depleting all tumor cell densities above a threshold of 0.2. The maximal chemotherapeutical dose is selected such that the upper value of $k^{-}$is of the order chosen in [17, 18, 19].

Figure 1 shows the starting point (when the tumor has been assessed by medical imaging), the time

\footnotetext{
${ }^{6}$ For a discussion about the timing of starting radiotherapy after resection see e.g., [36] and the references therein.
} 
point of resection (two weeks after start), and the result of surgical resection. Observe in Subfigure 1b the heterogeneous structure of the tumor, which corresponds to the known fact that glioma expand according to the brain structure, exhibiting a highly anisotropic behavior, as commented in Section 1. Due to this infiltrative growth, the resection is less successful at the tumor margins (Subfigure 1c).

Figure 2 shows the time point at the end of therapy in Strategies 2 and 3 (i.e., nine weeks after resection), follow-up pictures after two months for the different strategies, and scaled versions of these pictures. Notice the tumor recurrence which is more pronounced at the marginal region of the original neoplastic bulk. As expected, resection alone (first column in Figure 2) provides the poorest therapeutic outcome; the spread of glioma along white matter tracts of the brain tissue leads to the scattered shape of the tumor. This behavior is in line with clinically observed patterns (see e.g., [23, 57] and the references therein). Subsequent radiotherapy (middle column in Figure 2) is expected to enhance tumor eradication, and concurrent chemotherapy aiming at impairing cell-tissue adhesion by inhibiting receptor binding provides in our simulations an even better outcome (last column in Figure 2). However, complete eradication seems to be out of reach, as the scaled pictures in Subfigures 2b, 2d show. This is due to the high proliferation and migration ability of the glioma cells. We also compared adjuvant and neo-adjuvant chemotherapy (where the same chemotherapeutic agent was used) with surgery and radiotherapy, but the most significant differences were achieved with the strategies presented here in more detail. Whenever merely small improvements were obtained we opted for the less expensive and gentler strategy.

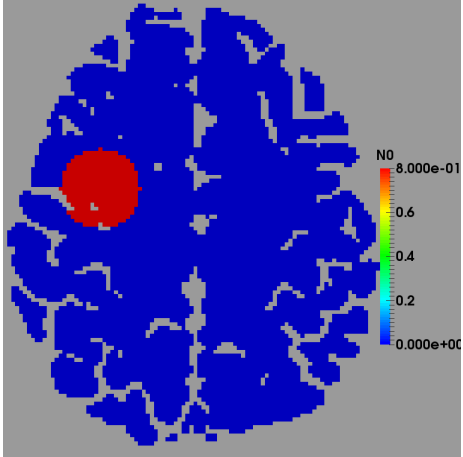

(a) Starting point

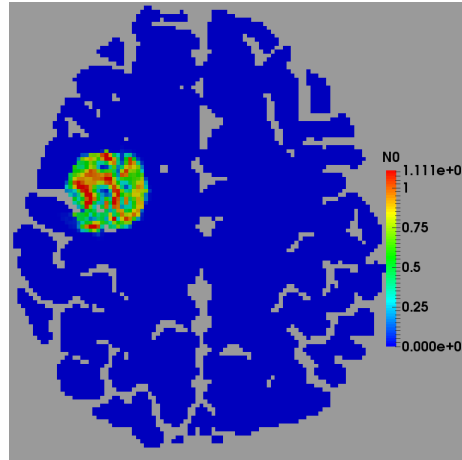

(b) Before resection

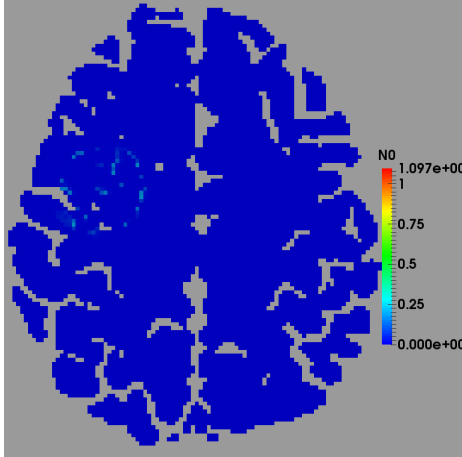

(c) After resection

Figure 1: Tumor at starting point, before resection, and after surgical resection.

\section{Conclusions and outlook}

In this note we started from previous multiscale models for glioma invasion and proposed some descriptions of therapy approaches, partly involving already standard surgery followed by radiotherapy, but also considering more recent therapeutic ideas connected to inhibition of cell-tissue attachment and its effects on migration and proliferation. Our multiscale setting with explicit subcellular dynamics seems particularly well suited to account for such features. Although we eventually work with the effective equations deduced on the macroscopic level, they carry in their coefficients the information from the lower (subcellular and individual cell) levels and assimilate DTI data allowing for a patient-specific description of the brain structure.

Several issues are yet to be addressed in future works; here we mention just a few: (i) When describing resection we set to zero all densities above 0.2 . While this is convenient to do in the computer, in clinical practice it is hardly possible to zoom (rescale) and assess the tumor heterogeneity at this level of detail; only the regions with high cell density can be observed by medical imaging and the tumor volume to be resected/irradiated (i.e., the CTV-PTV margin ${ }^{7}$ ) is established by following some general guidelines. However, our modeling approach opens the way for assessing the evolution of the tumor on the particular brain structure obtained by DTI and to account thereby for the infiltrative growth of glioma, which is a main factor in tumor recurrence. Hence, in forthcoming works more

\footnotetext{
${ }^{7} \mathrm{CTV}=$ clinical target volume, PTV=planning target volume
} 

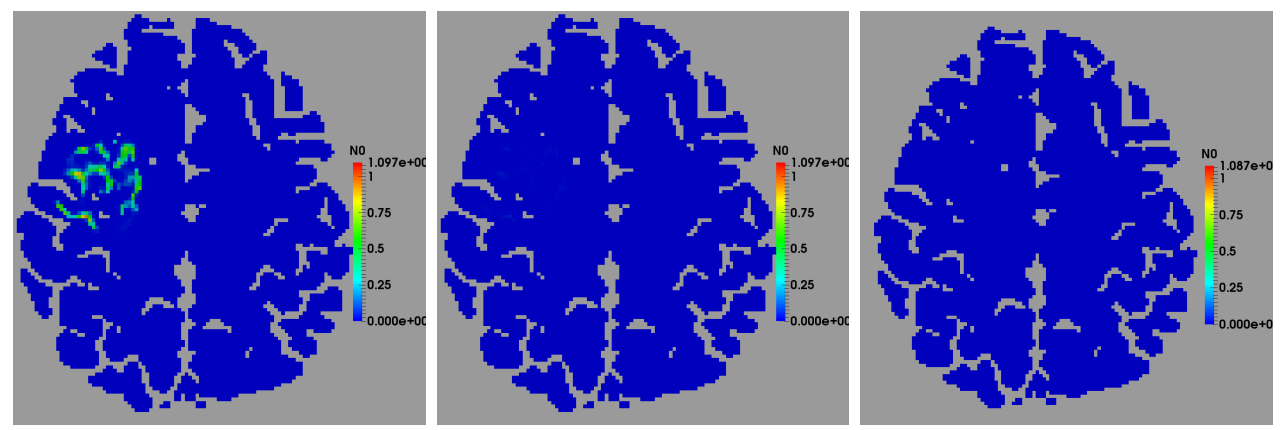

(a) End of therapy
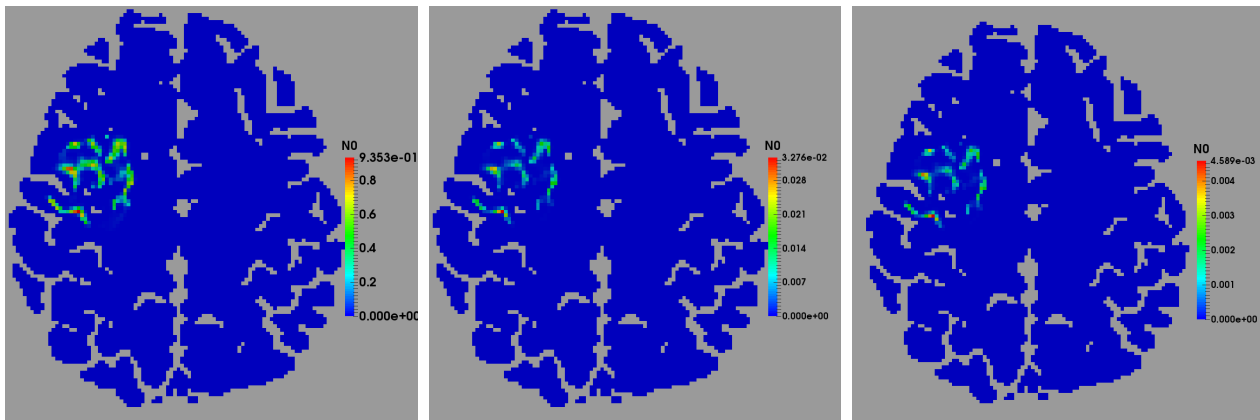

(b) End of therapy, scaled
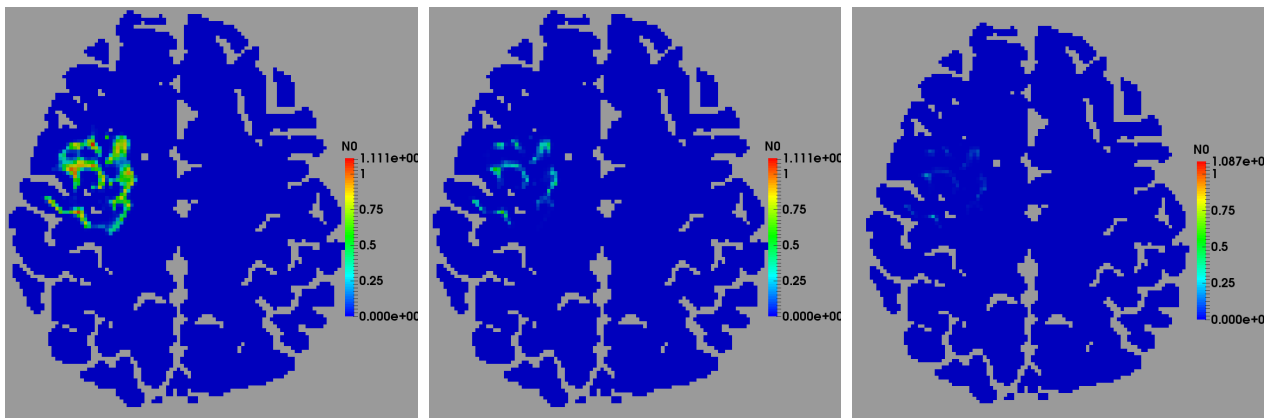

(c) Follow-up after two months
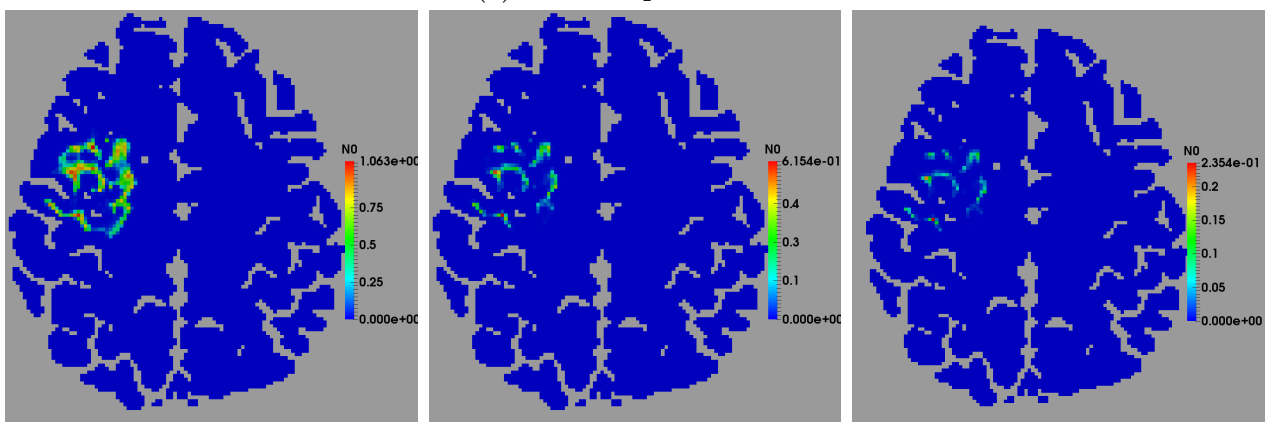

(d) Follow-up after two months, scaled

Figure 2: Comparison of Strategy 1 (left column), Strategy 2 (middle), and Strategy 3 (right).

attention will be paid to tumor delineation and treatment planning. (ii) Throughout the simulations we used the same DTI-assessed tissue structure via the functions $q$ and $Q$. Including an equation for characterizing the evolution of normal tissue would be desirable, but difficult to realize in this framework and with the available data, as the tissue structure would need to be assessed after each stage of therapy (resection and irradiation), which is expensive (if at all possible, at this level of detail). (iii) The parameters involved in our simulations have been taken from literature or empirically estimated. Determining them in a more precise way would mean to combine medical imaging techniques with biopsy and cell tracking; we refer e.g., to [49] for DTI image-guided biopsy studies. (iv) The effects 
of supplementary cell sensitization towards therapy and even enhancement of tumor cell degradation can be addressed by considering another chemotherapeutic agent, like temozolomide. It is one of the great advantages of mathematical modeling to be able to investigate a large variety of therapeutic approaches and to compare them.

\section{References}

[1] I Aganj, C. Lenglet, G. Sapiro, E Yacoub, K Ugurbil, and N Harel. Reconstruction of the orientation distribution function in single and multiple shell q-ball imaging with constant solid angle. Magnetic Resonance in Medicine, 64(2):554-566, 2010.

[2] A.L. Baldock, R.C. Rockne, A.D. Boone, M.L. Neal, and A. et al. Hawkins-Daarud. From patientspecific mathematical neuro-oncology to precision medicine. Frontiers in Oncology, 3:article 62, 2013.

[3] P. Bastian and M. Blatt. On the Generic Parallellisation of Iterative Solvers for the Finite Element Method. Int. J. Computational Science and Engineering, 4(1):56-69, 2008.

[4] P. Bastian, M. Blatt, A. Dedner, C. Engwer, R. Klöfkorn, R. Kornhuber, M. Ohlberger, and O. Sander. A generic grid interface for parallel and adaptive scientific computing. Part II: Implementation and tests in DUNE. Computing, 82(2-3):121-138, 2008.

[5] P. Bastian, M. Blatt, A. Dedner, C. Engwer, R. Klöfkorn, M. Ohlberger, and O. Sander. A Generic Grid Interface for Parallel and Adaptive Scientific Computing. Part I: Abstract Framework. Computing, 82(2-3):103-119, 2008.

[6] J. Besserer and U. Schneider. Track-event theory of cell survival with second-order repair. Radiation and Environmental Biophysics, pages 1-8, 2015.

[7] M. Blatt and P. Bastian. Lecture Notes in Scientific Computing, volume 4699. Springer, 2007.

[8] L. Bloy and R. Verma. On computing the underlying fiber directions from the diffusion orientation distribution function. In D. Metaxas, L. Axel, G. Fichtinger, and G. Székely, editors, Medical Image Computing and Computer-Assisted Intervention - MICCAI 2008, volume 5241 of Lecture Notes in Computer Science, pages 1-8. Springer Berlin Heidelberg, 2008.

[9] K. Böttger, H. Hatzikirou, A. Chauviere, and A. Deutsch. Investigation of the migration/proliferation dichotomy and its impact on avascular glioma invasion. Mathematical Modelling of Natural Phenomena, 7:105-135, 2012.

[10] D.J. Brenner, L.R. Hlatky, E.J. Hall, and R.K. Sachs. A convenient extension of the linearquadratic model to include redistribution and reoxigenation. Int. J. Rad. Onc. Biol. Phys., 32:379-390, 1995.

[11] M.C. Chamberlain, T. Cloughsey, D.A. Reardon, and P.Y. Wen. A novel treatment for glioblastoma. Expert Rev Neurother., 12:421-435, 2012.

[12] M. R. Chicoine and D. L. Silbergeld. Assessment of brain tumor cell motility in vivo and in vitro. Journal of Neurosurgery, 82(4):615-622, 1995.

[13] N. Cordes, J. Seidler, R. Durzok, H. Geinitz, and C. Brakebusch. Beta1-integrin-mediated signaling esentially contributes to cell survival after radiation-induced genotoxic injury. Oncogene, 25:1378-1390, 2006.

[14] G. D'Abaco and A. Kaye. Integrins: Molecular determinants of glioma invasion. J. of Clinical Neurosci., 14:1041-1048, 2007.

[15] T. Demuth and M.E. Berens. Molecular mechanisms of glioma cell invasion and migration. Journal of Neuro-Oncology, 70:217-228, 2004.

[16] J.S. Desgrosellier and D.A. Cheresh. Integrins in cancer: biological implications and therapeutic opportunities. Nat. Rev. Cancer, 10:9-22, 2010. 
[17] C. Engwer, T. Hillen, M. Knappitsch, and C. Surulescu. Glioma follow white matter tracts; a multiscale DTI-based model. J. of Math. Biol., 2014.

[18] C. Engwer, A. Hunt, and C. Surulescu. Effective equations for anisotropic glioma spread with proliferation: a multiscale approach and comparisons with previous settings. preprint, TU Kaiserslautern, 2014.

[19] C. Engwer, M. Knappitsch, and C. Surulescu. A multiscale model for glioma spread including cell-tissue interactions and proliferation. preprint, WWU Münster, 2014.

[20] R. Eymard, T. Gallouet, and R. Herbin. Finite Volume Methods. 2003.

[21] J. Fowler. The linear-quadratic formula and progress in fractionated radiotherapy. Br. J. Radiol., 62:679-694, 1989.

[22] F.B. Furnari, T. Fenton, R.M. Bachoo, A. Mukasa, J.M. Stommel, and A. et al. Stegh. Malignant astrocytic glioma: genetics, biology, and paths to treatment. Genes Dev., 21:2683-2710, 2007.

[23] A. Giese, R. Bjerkvig, M.E. Behrens, and M. Westphal. Cost of migration: invasion of malignant gliomas and implications for treatment. J. Clin. Oncol., 21(8):1624-1636, 2003.

[24] A. Giese, L. Kluwe, Meissner H., Michael E., and M. Westphal. Migration of human glioma cells on myelin. Neurosurgery, 38:755-764, 1996.

[25] A. Giese, M.A. Loo, N. Tran, D. Haskett, S. Coons, and M. Berens. Dichotomy of astrocytoma migration and proliferation. International Journal of Cancer, 67:275-282, 1996.

[26] A. Giese and M. Westphal. Glioma invasion in the central nervous system. Neurosurgery, 39:235$252,1996$.

[27] W. Guo and F.G. Giancotti. Integrin signaling during tumor progression. Nature Reviews, 5:816$826,2004$.

[28] E. Hall and A. Giaccia. Radiobiology for the radiologist. Lipincott Williams \& Wilkins, 2006.

[29] H. Hatzikirou, D. Basanta, M. Simon, K. Schaller, and A. Deutsch. 'go or grow': the key to the emergence of invasion in tumour progression? Math Med Biol, 29:49-65, 2012.

[30] T. Hillen. $\mathrm{M}^{5}$ mesoscopic and macroscopic models for mesenchymal motion. Journal of Mathematical Biology, 53:585-616, 2006.

[31] T. Hillen and K.J. Painter. Transport and anisotropic diffusion models for movement in oriented habitats. In M. Lewis, P. Maini, and S. Petrovskii, editors, Dispersal, Individual Movement and Spatial Ecology, volume 2071 of Lecture Notes in Mathematics, page 46. Springer, 2013.

[32] J.D. Hood and D.A. Cheresh. Role of integrins in cell invasion and migration. Nature Rev. Cancer, 2:91-100, 2002.

[33] A. Huttenlocher and A.R. Horwitz. Integrins in cell migration. Cold Spring Harbour Perspectives in Biology, 3:1-16, 2011.

[34] D.K. Jones, T.R. Knöschke, and R. Turner. White matter integrity, fiber count, and other fallacies: The do's and don'ts of diffusion MRI. NeuroImage, 73:239-254, 2013.

[35] J.P. Kirkpatrick, J.J. Meyer, and L.B. Marks. The linear-quadratic model is inappropriate to model high dose per fraction effects in radiosurgery. Semin. Rad. Oncol., 18:240-243, 2008.

[36] R. Lai, D.L. Hershman, T. Doan, and A.I. Neugut. The timing of cranial radiation in elderly patients with newly diagnosed glioblastoma multiforme. Neuro Oncol., 12:190-198, 2010.

[37] M.R. Lang, F.F. anf Gilbert. Diffusely infiltrative low-grade gliomas in adults. J. Clin. Oncol., 10:1236-1245, 2006.

[38] D.A. Lauffenburger and J.L. Lindermann. Receptors. Models for binding, trafficing and signaling. Oxford University Press, 1993. 
[39] F. Lefrank, J. Brotchi, and R. Kiss. Possible future issues in the treatment of glioblastomas: special emphasis on cell migration and the resistance of migrating glioblastoma cells to apoptosis. J. Clin. Oncol., 23:2411-2422, 2005.

[40] K.R. Legate, S.A. Wickström, and R. Fässler. Genetic and cell biological analysis of integrin outside-in signaling. Genes Dev., 23:397-418, 2009.

[41] T. Lorenz and C. Surulescu. On a class of multiscale cancer cell migration models: Well-posedness in less regular function spaces. Math. Models Meth. Appl. Sci., 24, 2014. 54 pages.

[42] T. Mangum and I. Nakano. Glioma stem cells and their therapy resistance. J. Carcinogene Mutagene, S1-002:1-8, 2011.

[43] K.V. Mardia and P.E. Jupp. Directional Statistics. Wiley, 1999.

[44] G. Meral, C. Stinner, and C. Surulescu. A multiscale model for acid-mediated tumor invasion: therapy approaches. Preprint, TU Kaiserslautern (2014), submitted.

[45] S.J. Moschos, L.M. Drogowski, S.I. Reppert, and J.M. Kirkwood. Integrins and cancer. Oncology, 21(9):13-20, 2007.

[46] N. Oppenheimer-Marks and P.E. Lipsky. Adhesion molecules and the regulation of the migration of lymphocytes. In A. Hamann, editor, Adhesion Molecules and Chemokynes in Lymphocyte Trafficking, pages 55-88. Harwood Acad. Publ., Amsterdam, 1997.

[47] K.J. Painter and T. Hillen. Mathematical modelling of glioma growth: the use of diffusion tensor imaging (DTI) data to predict the anisotropic pathways of cancer invasion. Journal for Theoretical Biology, 323:25-39, 2013.

[48] M. Preusser, S. de Ribaupierre, and A. et al. Wohrer. Current concepts and management of glioblastoma. Ann. Neurol., 70:9-21, 2011.

[49] A.J. Price, R. Jena, N.G. Burnet, P.J. Hutchinson, A.F. Dean, A. Peña, J.D. Pickard, T.A. Carpenter, and J.H. Gillard. Improved delineation of glioma margins and regions of infiltration with the use of diffusion tensor imaging: An image-guided biopsy study. Am. J. Neuroradiol., 27:1969-1974, 2006.

[50] M. Reiser, F.-P. Kuhn, and J. Debus. Radiologie. Thieme, 2011.

[51] R. Rockne, E.C. Alvord Jr., J.K. Rockhill, and K.R. Swanson. A mathematical model for brain tumor response to radiation therapy. J. Math. Biol., 58:561-578, 2009.

[52] R.C. Rockne, A.D. Trister, J. Jacobs, A.J. Hawkins-Daarud, M.L. Neal, and K. et al. Hendrickson. A patient-specific computational model of hypoxia-modulated radiation resistance in glioblastoma using 18f-fmiso-pet. J. R. Soc. Interface, 12:1-10, 2015.

[53] M. Ruzicka. Nichtlineare Funktionalanalysis. Eine Einführung. Springer, 2004.

[54] R.K. Sachs and D.J. Brenner. The mechanistic basis of the linear-quadratic model. Med. Phys., 25:2071-2073, 1998.

[55] C. Sanderson. Armadillo: An Open Source C ++ Linear Algebra Library for Fast Prototyping and Computationally Intensive Experiments. Technical Report,NICTA, 2010.

[56] S. Sathornsumetee, D.A. Reardon, A. Desjardins, J.A. Quinn, J.J. Vredenburgh, and J.N. Rich. Molecularly targeted therapy for malignant glioma. Cancer, 110:13-24, 2007.

[57] E. (ed.) Scarabino. Imaging Gliomas after Treatment. A Case-based Atlas. Springer, 2012.

[58] R.E. Showalter. Monotone Operators in Banach Space and Nonlinear Partial Differential Equations. AMS, 1997.

[59] G.G. Steel. Basic Clinical Radiobiology. Arnold, 2002. 
[60] J. Steinbach and M. Weller. Apoptosis in gliomas: molecular mechanisms and therapeutic implications. J. NeuroOncol., 70:245-254, 2004.

[61] C. Stinner, C. Surulescu, and G. Meral. A multiscale model for ph-tactic invasion with timevarying carrying capacities. IMA J. of Appl. Math., doi:10.1093/imamat/hxu055, 2014.

[62] R. Stupp and C. Ruegg. Integrin inhibitors reaching the clinic. J. Clin. Oncol., 25:1637-1638, 2007.

[63] J.H. Uhm, C.L. Gladson, and J.S. Rao. The role of integrins in the malignant phenotype of gliomas. Frontiers in Bioscience, 4:188-199, 1999.

[64] S. Wagner, S.M. Rampersad, Ü. Aydin, J. Vorwerk, T.F. Oostendorp, T. Neuling, C.S. Herrmann, D.F. Stegeman, and C.H. Wolters. Investigation of tDCS volume conduction effects in a highly realistic head model. Journal of neural engineering, 11(1):016002, 2014.

[65] B.G. Woulters and J.M. Brown. Cells at intermediate oxygen levels can be more important than the hypoxic fraction in determining tumor response to fractionated radiotherapy. Radiat. Res., 147:541-, 1997.

[66] L. Zhan, A.D. Leow, S. Zhu, M. Barysheva, A.W. Toga, K.L. McMahon, G.I. de Zubicaray, M.J. Wright, and P.M. Thompson. A novel measure of fractional anisotropy based on the tensor distribution function. In G.-Z. Yang, D. Hawkes, D. Rueckert, A. Noble, and C. Taylor, editors, Medical Image Computing and Computer-Assisted Intervention - MICCAI 2009, volume 5761 of Lecture Notes in Computer Science, pages 845-852. Springer Berlin Heidelberg, 2009. 